\title{
Extraction and Recrystallization of Mesoionic Pyrimidinium Betaines
}

\author{
Fatiha Malki, Abdelkader Touati, and Saâd Moulay
}

\begin{abstract}
Monocyclic, bicyclic and fatty chain mesoionic pyrimidinium betaines were synthesized and separated from other compounds by liquid-liquid extraction method and then recrystallized from acetone or diethyl ether. The thus-purified betaines were characterized by spectroscopic analyses, including UV-visible, IR, ${ }^{1} \mathrm{H}-\mathrm{NMR}$ and MS.
\end{abstract}

Index Terms-Betaine, extraction, pyrimidine,
recrystallization.

\section{INTRODUCTION}

The term "betaine" is commonly used to refer to the glycine betaine ( $N, N, N$-trimethylglycine), but also to its derivatives and to any organic compound carrying a quaternary nitrogen [1].

Betaine is present in various plants, animals and microorganisms and plays an important role in osmotic stress resistance in these organisms. This molecule is a human nutrient and is used in the treatment of various diseases such as homocystinuria and homocystenemia cardiovascular diseases, depression and other mental disorders, seizures and arthritis [2].

Alkylbetaines represent a class of zwitterionic surfactants, which are electro neutral inner salts in a wide $\mathrm{pH}$ range with a positive charge on the nitrogen atom and a negative one on the carboxyl group [3]. They constitute a group of products of increasing importance in cosmetic, household and industrial applications [4]. They have a variety of uses in medicine, pharmacy, biology and other scientific fields [5]. They also find industrial applications as softening and wetting agents in textile production (mainly due to their antistatic and softening properties) and as emulsifiers and dispersants, for example, for photographic emulsions [3].

In order to exploit its numerous effects, we have synthesized heterocyclic mesoionic compounds with a pyrimidine ring in their structures. These chemical compounds present interesting biological activities [6].

Mesoionic pyrimidinium betaines are mesomeric compounds whose positive and negative charges are exclusively restricted to separate parts of the molecules having $\pi$-electron system [7]. Heterocyclic mesomeric

Manuscript received September 4, 2013; revised November 23, 2013 This work was supported by research funding from Ecole Normale Supérieure.

F. Malki and A. Touati are with the Laboratoire de recherche sur les produits bioactifs et valorisation de la biomasse, Ecole Normale Supérieure, BP 92,16000, Kouba, Algiers, Algéria (e-mail: malki_fatiha76@yahoo.fr a_touati2000@yahoo.fr).

S. Moulay is with the Laboratoire de Chimie-Physique Moléculaire et Macromoléculaire, Département de Chimie Industrielle, Faculté des Sciences de L'Ingénieur, Université Saâd Dahlab de Blida, Blida, Algeria (e-mail: polymchemlab@hotmail.com). betaines not only are interesting starting materials for the synthesis of bio inspired heterocyclics, but also form a class of natural products by themselves [8].

Pyrimidinium betaines have been studied by several authors for their particular structure [9], their biological and pharmacological activity [10], [11], their chemical stability [12], and their proneness to cycloaddition reaction[13], [14]. Numerous methods of synthesis of betaines bearing pyrimidine ring were reported.

Betaines with monocyclic and bicyclic structures 5 and 6 were prepared by condensation of $\alpha$-aminopyridine 1 or the amidine derivatives 2 with the appropriate malonic acid derivatives 3 [15]-[17], or with the carbon suboxide 4 [14]. See Fig. 1.

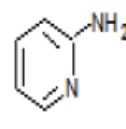

1<smiles>[R]N=C([R])N[R]</smiles>

2<smiles>[R]c1c([O-])[n+]([R])c([R])n([R])c1=O</smiles>

5<smiles>[R20][R]([R20])C([R])C([R])[R]</smiles>

3

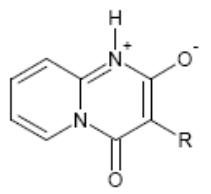

6
Fig. 1. Synthetic reaction for betaine 6 .

Other methods of synthesis of pyrimidinium betaines consist in reacting the derivatives of $N, N^{\prime}$-disubstituted amidine with substituted malonic acid and dicyclohexylcarbodiimide (DCC) as a condensing agent at room temperature, to yield the mesoionic structures with an extended conjugated $\pi$-system and carbon framework [7].

In this work, we were interested in the synthesis of mesoionic pyrimidinium betaines with monocyclic and bicyclic structures, as well as fatty chain betaines. These chemical compounds would possess biological activity due to the presence of the pyrimidine ring [18], and surface active properties owing to their surfactant structure.

Analysis of our betaines by thin layer chromatography (TLC) and UV-visible spectrophotometry revealed a mixture of compounds of different chemical natures. Besides, the application of the conditions cited in the literature [17] did not allow us to obtain pure betaines. Therefore, liquid-liquid extraction was valorized as a useful technique to isolate the betaines, providing that all components of the mixture were soluble in organic solvents and insoluble in water, and that the acidic and the basic compounds could be moved in their salt forms into the aqueous phase. 


\section{MATERIAL AND METHODS}

In this study, thin layer chromatography plates (TLC) were made of silica gel 60 F254 (Merck). The elution was carried out by a mixture of solvents and the different spots were visualized using UV lamp at $254 \mathrm{~nm}$. UV-visible analysis was conducted using a Shimadzu 1605 double beam spectrophotometer. The used chemicals were of analytical grade purity and were obtained from either Sigma-Aldrich or Merck.

\section{A. Synthesis of Bicyclic Pyrimidinium Betaines}

The bicyclic betaine 9 was synthesized according to the method of Huhn et al. [17] by condensation of $\alpha$-aminopyridine 8 with the bispentachlorophenyl ester of phenyl malonic acid 7 (Fig. 2). The reaction was realized in acetone as an aprotic solvent at room temperature and in a few minutes the product precipitated. After filtration work-up, the bicyclic betaine 9 was obtained in form of yellow solid in a yield of $72.5 \%$.

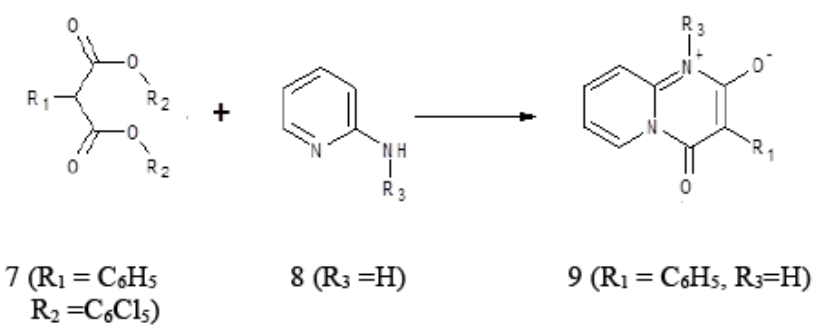

Fig. 2. Synthetic reaction for betaine 9 .

\section{B. Synthesis of Monocyclic Pyrimidinium Betaines}

The monocyclic betaine 11 was prepared by condensation of malonic ester 7 with $N, N$ '-diphenylbenzamidine 10 [17], [19] in diethyl ether at room temperature (Fig. 3). The monocyclic pyrimidinium betaine 11 was obtained as yellow solid in a yield of $66 \%$ and then was recrystallized in acetone. Under identical protocol, fatty chain pyrimidinium betaine 13 was made in $58.7 \%$ [19].

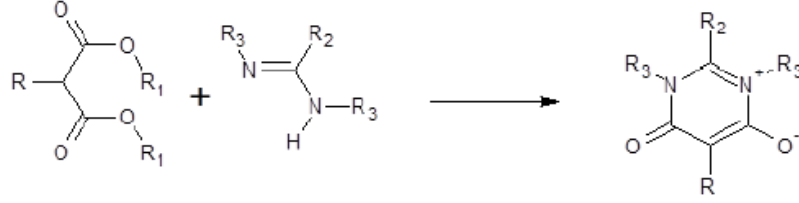

$$
\begin{aligned}
& \begin{array}{rlr}
7 \underset{\mathrm{R}=\mathrm{C}_{6} \mathrm{H}_{5},}{\mathrm{R}_{1}=\mathrm{C}_{6} \mathrm{Cl}_{5}} & 10\left(\mathrm{R}_{2}=\mathrm{R}_{3}=\mathrm{C}_{6} \mathrm{H}_{5}\right) & 11\left(\mathrm{R}=\mathrm{R}_{2}=\mathrm{R}_{3}=\mathrm{C}_{6} \mathrm{H}_{5}\right) \\
& 12\left(\mathrm{R}_{2}=\mathrm{C}_{11} \mathrm{H}_{23},\right. & 13\left(\mathrm{R}_{2}=\mathrm{C}_{11} \mathrm{H}_{23},\right. \\
\left.\mathrm{R}_{3}=\mathrm{C}_{6} \mathrm{H}_{5}\right) & \left.\mathrm{R}=\mathrm{R}_{3}=\mathrm{C}_{6} \mathrm{H}_{5}\right)
\end{array}
\end{aligned}
$$

Fig. 3. Synthetic reaction for monocyclic betaines 11 and 13 .

\section{Extration Procedure}

The mixture obtained formed in A and B was dissolved in dichloromethane (DCM) and the organic solution was then treated sequentially with $10 \% \mathrm{HCl}$ and $10 \% \quad \mathrm{Na}_{2} \mathrm{CO}_{3}$. Afterwards, the malonic ester was extracted as sodium carboxylate, pentachlorophenol (side product) as sodium phenolate, and $\alpha$-aminopyridine as well as amidine as ammonium hydrochlorides. Betaine, alone and as neutral compound, remained in DCM layer. Fig. 4 illustrates the scheme used for the separation by liquid-liquid extraction of different components of a mixture.

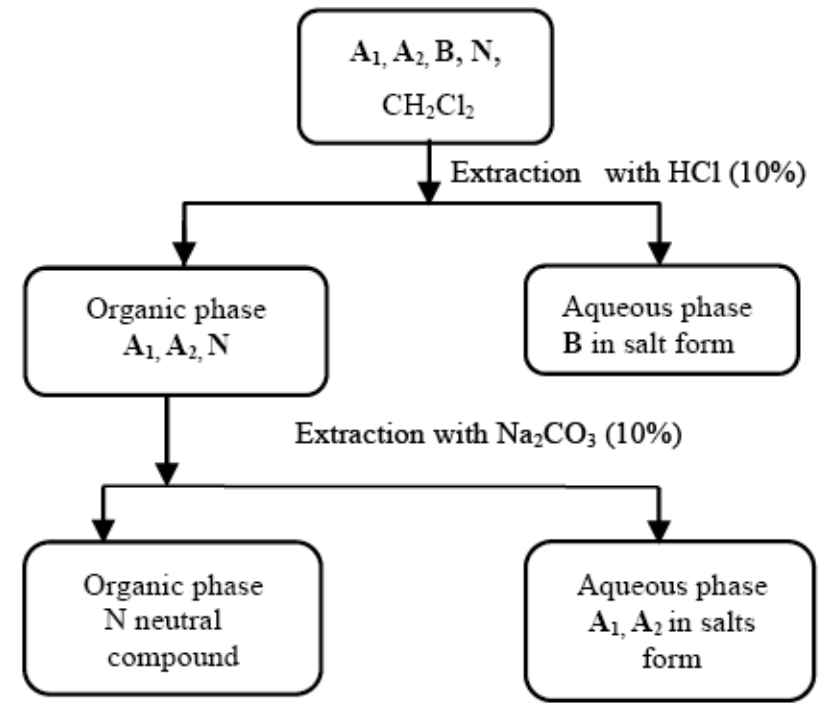

$A_{1}$ : a derivative of the carboxylic acid such as malonic ester 7 $\mathrm{A}_{2}$ : a weak acid such as pentachlorophenol (side product) $\mathrm{B}$ : a base such as $\alpha$-aminopyridine 8 or amidines 10,12 $\mathrm{N}$ : a neutral compound such as, betaine $9,11,13$.

Fig. 4. Schema for separation by liquid-liquid extraction of the components of a mixture.

\section{RESULTS AND DISCUSSION}

Analysis of the synthesized betaines by TLC showed the presence of four spots corresponding to four different compounds: $\alpha$-aminopyridine or amidine, malonic ester, betaine and pentachlorophenol (PCP). Betaines were separated from the liquid mixture after several successive liquid-liquid extractions. Table I and Table II summarize the number of extractions used for separation of the $\alpha$-aminopyridine, amidine, malonic ester and pentachlorophenol from the mixture.

TABLE I: NUMBER OF EXTRACTIONS USED FOR THE SEPARATION OF EACH COMPOUND FROM THE MIXTURE IN THE CASE OF BICYCLIC BETAINE 9 AND

\begin{tabular}{|l|l|c|c|}
\hline \multicolumn{2}{|c|}{ MonOCYLIC BETAINE 11 } & Number of \\
& \multicolumn{1}{|c|}{ Compounds } & 3 & 5 \\
\hline $\mathrm{HCl}(10 \%)$ & $\alpha$-Aminopyridine & - & + \\
$\mathrm{HCl}(10 \%)$ & Amidine & - & + \\
$\mathrm{Na}_{2} \mathrm{CO}_{3}(10 \%)$ & Malonic ester & - & + \\
$\mathrm{Na}_{2} \mathrm{CO}_{3}(10 \%)$ & Pentachlorophenol & + & + \\
& & & \\
\hline
\end{tabular}

+: Disappearance of the compound from the organic phase

-: No disappearance of the compound from the organic phase

TLC and UV-Visible analyses of the organic phase after each extraction allowed us to assess the number of extractions with $\mathrm{HCl}$ and $\mathrm{Na}_{2} \mathrm{CO}_{3}$ solutions required for the separation of each component of the mixture. And as shown in Table I, the bicyclic and monocyclic betaines were separated after five extractions. In the case of fatty chain betaine, TLC and UV-Visible analyses suggested seven extractions for the isolation of the fatty chain amidine and PCP from the organic phase. Hence, malonic ester and fatty 
chain betaine remained in the organic phase.

TABLE II: NUMBER OF EXTRACTIONS USED FOR THE SEPARATION OF EACH Compound From the MiXTURE In the CASE OF FATTy Chain Betaine13

\begin{tabular}{|l|l|c|c|}
\hline Solutions & \multicolumn{1}{|c|}{ Number of } \\
& Compounds & 5 & 7 \\
\hline $\mathrm{HCl}(10 \%)$ & Fatty chain amidine & - & + \\
$\mathrm{Na}_{2} \mathrm{CO}_{3}(10 \%)$ & Malonic ester & - & - \\
$\mathrm{Na}_{2} \mathrm{CO}_{3}(10 \%)$ & Pentachrophenol & - & + \\
\hline
\end{tabular}

-: no disappearance of the compound from the organic phase

We noted that malonic ester still remained in the organic phase, the fact that prompted us to replace the basic solution $\mathrm{Na}_{2} \mathrm{CO}_{3}(10 \%)$ by stronger alkaline solutions such as alcoholic or aqueous $\mathrm{KOH}(10 \%)$. However, this basic hydrolysis reaction led to the following results:

- No disappearance of the ester.

- Occurrence of a saponification reaction.

- Skipping of the fatty chain betaine into aqueous phase.

In another experiment, the mixture (betaine, malonic ester and $\mathrm{NaOH}(10 \%)$ ) in DCM was heated to reflux for $1 \mathrm{~h}$. After extraction, the betaine was obtained in a very pure state, but in lower yield. The experiment was repeated by adding first a solution of $\mathrm{Na}_{2} \mathrm{CO}_{3}(10 \%)$ to extract PCP, then the organic phase containing malonic ester, betaine, $\mathrm{Na}_{2} \mathrm{CO}_{3}(10 \%)$ and DCM, was refluxed for $1 \mathrm{~h}$. From this work-up and after extraction, the fatty chain betaine could be isolated in a high purity. However, when $\mathrm{NaOH}(10 \%)$ was used and without heating, the fatty chain betaine was separated in better yield and higher purity after extraction and recrystallization from diethyl ether or acetone. Table III summarizes the physical characteristics and the reaction yields of the pure synthesized betaines.

By comparing the reaction yields of betaines shown in Table III, we noted the decrease of the yields after purification, especially in the case of fatty chain betaine. This is due, on the one hand to the number of extractions used which has led to the loss of a great part of this product, and, on the other hand, to the fact that the reagents did not fully react. The yield was enhanced when an alkaline solution $\mathrm{NaOH}(10 \%)$ was employed to neutralize the derivatives of malonic acid.

The betaines synthesized were characterized by spectroscopic analyses, UV-visible, IR, ${ }^{1} \mathrm{H}-\mathrm{NMR}$ and MS.

\section{Spectral characterizations of the synthesized betaines}

9:UV(1,4-dioxane), $\lambda_{\max }[\mathrm{nm}](\log \varepsilon)=355.5(3.68) ; \mathrm{IR}$ $(\mathrm{KBr}),\left[\mathrm{cm}^{-1}\right]=3112($ ar. $\mathrm{C}-\mathrm{H}), 1678(\mathrm{C}=\mathrm{O}), 1527$ (arom. $\mathrm{C}=\mathrm{C}$ ); ${ }^{1} \mathrm{H}-\mathrm{NMR}$ (DMSO-d ${ }_{6}$ ), $\delta[\mathrm{ppm}]=7.2-7.9$ (arom. $-\mathrm{H}$ ), $9.16(6-\mathrm{H}), 8.16(8-\mathrm{H}) ; \mathrm{MS}, \mathrm{m} / \mathrm{z}=238$.

11: UV(1,4-dioxane), $\lambda_{\max }[\mathrm{nm}](\log \varepsilon)=357.5(3.07)$; $\mathrm{IR}(\mathrm{KBr}),\left[\mathrm{cm}^{-1}\right]=3051$ (arom. C-H), $1651(\mathrm{C}=\mathrm{O}), 1593$ (arom. $\mathrm{C}=\mathrm{C}) ;{ }^{1} \mathrm{H}-\mathrm{NMR}\left(\mathrm{DMSO}_{\mathrm{d}}\right), \delta[\mathrm{ppm}]=6.9-7.8($ arom. $-\mathrm{H})$; $\mathrm{MS}, \mathrm{m} / \mathrm{z}=416$.

13: UV(1,4-dioxane), $\lambda_{\max }[\mathrm{nm}](\log \varepsilon)=352(3.43) ; \mathrm{IR}$ $(\mathrm{KBr})$,

$\left[\mathrm{cm}^{-1}\right]=3052$ (arom. C-H), $1650(\mathrm{C}=\mathrm{O}), 1597$ (arom. $\mathrm{C}=\mathrm{C}) ;{ }^{1} \mathrm{H}-\mathrm{NMR}\left(\mathrm{CDCl}_{3}\right), \delta[\mathrm{ppm}]=7.23($ arom. $-\mathrm{H}), 2.38 \mathrm{H}$ $\left.\left(\alpha-\mathrm{CH}_{2}\right), 1.27 \mathrm{H}\left(-\mathrm{CH}_{2}\right)_{9}\right), 0.89 \mathrm{H}\left(-\mathrm{CH}_{3}\right)$.
TABLE III: PHYSICAL CHARACTERIZATION OF SYNTHESIZED BETAINES

\begin{tabular}{|lllll|}
\hline \multirow{2}{*}{ Betaine } & Aspect & m.p. $\left({ }^{\circ} \mathrm{C}\right)$ & \multicolumn{2}{c|}{ Yield $(\%)$} \\
& & & $\mathrm{Y}_{1}{ }^{\mathrm{a}}$ & $\mathrm{Y}_{2}{ }^{\mathrm{b}}$ \\
\hline & & & & \\
11 & Yellow crystals & $320.0-312.0^{\mathrm{c}}$ & 72.5 & 69.0 \\
13 & Yellow crystals & $317.0-319.0^{\mathrm{c}}$ & 66.0 & 46.3 \\
& White needles & $124.1-127.9$ & 58.7 & $18.0^{\mathrm{d}}$ \\
& & & & $30.0^{\mathrm{e}}$ \\
& & & & $37^{\mathrm{f}}$ \\
\hline
\end{tabular}

a) Before purification; b) After purification; c) With decomposition; d) $10 \%$ $\mathrm{NaOH}$ and under reflux; e) $10 \% \mathrm{Na}_{\hat{e}} \mathrm{CO}_{3}$ and under reflux; f) $10 \% \mathrm{NaOH}$ and no reflux

\section{CONCLUSION}

Mesoionic pyrimidinium betaines could be prepared in good yields under mild conditions. Liquid-liquid extractions with aqueous acidic and basic solutions enabled effective separation of the betaines in high purity from their mixtures components. Yet, an excessive extraction may lower the yield as in the case of fatty chain betaine.

\section{REFERENCES}

[1] Z. F. Nsimba, M. Paquot, L. G. Mvumbi, and M. Deleu, "Les dérivés tensioactifs de la glycine bétaïne: méthodes de synthèse et potentialités d'utilisation," Biotechnol. Agron. Soc. Environ., vol. 14, no. 04, pp. 737-748, 2010.

[2] F. N. Zakanda, P. Laurent, M. Paquot, G. M. Lelo, and M. Deleu, "Alkylbetainate chlorides: Synthesis and behavior of monolayers at the air-water interface," Thin Solid Films, vol. 520, no. 01, pp. 344-350, 2011.

[3] N. C. Christov, N. D. Denkov, P. A. Kralchevsky, K. P. Ananthapadmanabhan, and A. Lips, "Synergistic sphere-to-rod micelle transition in mixed solutions of sodium dodecyl sulfate and cocoamidopropyl betaine," Langmuir, vol. 20, no. 03, pp. 565-571, 2004.

[4] M. A. Gonzalez, J. C. Royero, A. Mesa, and L. B. Galvis, "Synthesis and biological evaluation of pyridine betaine A and B," Natl. Prod. Res., vol. 23, no. 16, pp. 1485-1491, 2009.

[5] X. Domingo, "Betaines," in E. G. Lomax Ed., Amphoteric Surfactants, New York: Marcel Dekker 1996, pp.75-190.

[6] F. Malki and A. Touati, "Evaluation of antioxidantactivity of heterocyclic mesomeric betaines containing pyrimidine moiety," in Proc. Malaysia International Biological Symposium 2012, i-Simbiomas 2012, Selangor, Malaysia, July 2012, pp. 11-12.

[7] A. Koch, U. Jonas, H. Ritterb, and H.-W. Spiess, "Extended mesoionic systems: synthesis and characterization of monocyclic, polycyclic and macrocyclicpyrimidinium-olate derivatives and their photochemical behavior," Tetrahedron, vol. 60, no. 44, pp. 10011-10018, 2004.

[8] A. S. Lindner, M. Nieger, and A. Schmidt, "Synthesis and properties of imidazo[1,2-a]pyridinium-3-olate. Some revised structures," Tetrahedron, vol. 65, no. 36, pp. 7591-7596, 2009.

[9] C. Kratky and T. Kappe, "Mesoionic six-membered heterocycles. XIV. Crystal structure of a pyrimidine betaine," J. Heterocycl. Chem., vol. 18, no. 05, pp. 881-883, 1981.

[10] R. A. Coburn and R. A. Carapellotti, "Synthesis and properties of mesoionic pyrimido [1, 2-b] pyridazine-2, 4-diones and mesoionic pyridazino [2, 3-a]-s-triazine-2, 4-diones: Mesoionic analogs structurally related to fervenulin," J. Pharm. Sci., vol. 65, no. 08, pp. 1505-1510, 1976.

[11] R. A. Glennon, R. G. Bass, and E. Schubert, "Alkylation study on 6-ethyl-2, 3-dihydrothiazolo-[3, 2a] pyrimidine-5, 7-diones,” $J$. Heterocycl. Chem., vol. 16, no. 05, pp. 903-907, 1979.

[12] G. Wenska, M. Insińska, and B. Skalski, "Synthesis and solvatochromism of 2-(N-pyridinio)-pyrimidin-4-olate and related betaines derived from uracils," Polish J. Chem., vol. 74, pp. 659-672, 2000 .

[13] T. Kappe and W. Lube, "Cycloadditions with mesomeric pyrimidine betaines," Angew. Chem. Int. Ed. Engl., vol.10, no. 12, pp. 925-926, 1971.

[14] K. T. Potts and M. Sorm, "Mesoionic compounds XIII. 1, a (4 Dipolar-type cycloaddition reactions of anhydro-2-hydroxy-1-methyl-4-oxopyrido [1, 2-a] pyrimidinium hydroxide," J. Org. Chem., vol. 36, no. 01, pp. 8-10, 1971. b) ibid, 
"Mesoionic compounds. XVI. 1,4-Dipolar type cycloaddition reactions utilizing pyrimidinium betaines," J. Org. Chem., vol. 37, no. 09, pp. 1422-1425, 1972.

[15] A. E. Tschitschibabin, "Formation of bicyclic derivatives of $\alpha$-aminopyridine," Ber. Dtsch .chem. Ges., vol. 57, pp. 1168-1172, 1924.

[16] T. Kappe and W. Lube, "Zur Synthese MesomererPyrimidinbetaine," Monatsh. Chem, vol. 102, pp. 781-787, 1971.

[17] P. Dvortsak, G. Resofszki, M. Huhn, L. Zalantai, and A. I. Kiss, "Reactions of pentachlorophenyl esters of malonic acid derivatives-II Preparation and investigation of pyrimidine betaines," Tetrahedron, vol. 32, no. 17, pp. 2117-2120, 1976.

[18] T. A. Naik and K. H.Chikhalia, "Studies on synthesis of pyrimidine derivatives and their pharmacological evaluation," E-Journal of Chemistry, vol. 4, no. 1, pp. 60-66, 2007.

[19] F. Malki, A. Touati, S. Rahal, and S. Moulay, "Total synthesis of monocyclic pyrimidinium betaines with fatty alkyl chain," Asian $J$. Chem., vol. 23, no. 3, pp. 961-967, 2011.

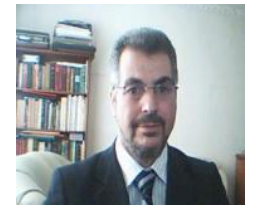

Saâd Moulay is an Algerian citizen, received his bachelor's degree in chemistry from the Université des Sciences et Technologie Houari Boumèdiène USTHB (Algiers, Algeria) in 1979. He then received his $\mathrm{Ph} . \mathrm{D}$. degree in organic and polymer chemistry, under the supervision of Emeritus Professor William H. Daly, from the chemistry department of Louisiana State University (Baton Rouge, La, USA) in 1986. He is currently Professor of chemistry at Département de Chimie Industrielle of Université Saad Dahlab de Blida (Blida, Algeria).

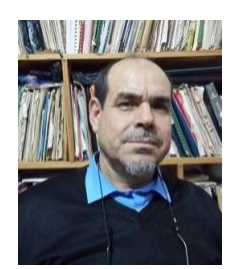

Abdelkader Touati was born in Algeria at Boumerdes on march 3, 1959. He received his bachelor's degree in chemistry from the Université des Sciences et Technologie Houari Boumèdiène USTHB (Algiers, Algeria) in 1984. He then received his Ph.D. degree in molecular organic chemistry from Paul Sabatier University (Toulouse, France) in 1988. He is currently Professor and researcher in chemistry at Département (Algiers, Algeria) de Chimie of Ecole Normale Supérieure de Kouba 